\title{
The Radar Cross Section Analysis of Radome Based on Conjugate Gradient Fast Fourier Transform
}

\author{
Huan Yu, Hao Gu, Bing Liu, Kang Fan, Hanming Gu, Yingkai Zhou, Yanjiao Ge \\ Shanghai Radio Equipment Research Institute, Shanghai, China \\ Email: yuh668@126.com
}

How to cite this paper: $\mathrm{Yu}, \mathrm{H}$., Gu, H., Liu, B., Fan, K., Gu, H.M., Zhou, Y.K. and Ge, Y.J. (2020) The Radar Cross Section Analysis of Radome Based on Conjugate Gradient Fast Fourier Transform. Journal of Applied Mathematics and Physics, 8, 933-940.

https://doi.org/10.4236/jamp.2020.85072

Received: March 16, 2020

Accepted: May 24, 2020

Published: May 27, 2020

\begin{abstract}
This paper presents an algorithm for analysis of the dielectric radomes. In this method, the radome is discretized by a regular grid with rooftop basic functions. The Volume Integral Equation (VIE) for 3D dielectric object is transformed to linear system by Galerkin's testing formulation. Furthermore, the linear system is presented by Toeplitz matrix which can be solved by the Conjugate Gradient algorithm combined with Fast Fourier Transform (CG-FFT) iteratively. Also, the algorithm requires less computational complexity and memory. This paper simulates the mono-static Radar Cross Section of dielectric radome by the CG-FFT, which was validated against commercial software FEKO.
\end{abstract}

\section{Keywords}

Radome, Radar Cross Section, Volume Integral Equation, CG-FFT

\section{Introduction}

Radome is a significance component in modern communication system. Commonly, radome consists of dielectric materials [1] [2] [3] [4]. Modeling and efficient simulation of radome plays an important role in analysis. Computational Electromagnetics (CEM) is a method for electromagnetic analysis of the radome. Commonly, Finite Element Method (FEM) Finite Difference Time Domain (FDTD) and Method of Moment (MoM) are popular methods of CEM. However, for the simulation of radome, FEM and FDTD usually consume a lot of memory because of its discretization of whole region including the volume which is vacuum [5]. MoM only requires the discretization of dielectric object rather than the whole region, but has to solve the linear equation, which means 
that it consumes computational complexity $\mathrm{O}\left(\mathrm{N}^{2}\right)$ if solved iteratively [6] [7].

In this paper, we present an algorithm called CG-FFT for the simulation of dielectric radome, which applies Fast Fourier Transform combined with Conjugate Gradient algorithm. And it requires less computational complexity if solved iteratively. We also calculate the mono-static RCS of a dielectric radome to validate the algorithm. Generally, CG-FFT is a feasible method for the simulation of dielectric radome.

\section{Theory}

The governing equation for three-dimensional dielectric object is following [8]:

$$
\mathbf{E}^{i}(r)=\frac{\mathbf{D}\left(r^{\prime}\right)}{\varepsilon(r)}-\left(k^{2}+\nabla \nabla \cdot\right) \mathbf{A}(r)
$$

where the $\mathbf{E}^{i}(r)$ is the incident electric field, the wave number $k=\omega\left(\mu_{0} \varepsilon_{0}\right)^{1 / 2}, \omega$ is angular frequency. $\mathcal{E}_{0}$ is dielectric constant in vacuum, $\mu_{0}$ is permeability constant in vacuum, $\varepsilon(\boldsymbol{r})$ is the relative dielectric distribution function in dielectric object. And $\boldsymbol{r}$ is the observation location, $\boldsymbol{r}^{\prime}$ represents the source location. $\mathbf{D}\left(r^{\prime}\right)$ represents the electric displacement field. Field $\mathbf{A}(r)$ is following:

$$
\mathbf{A}(r)=\frac{1}{\varepsilon_{0}} \iiint_{V} G\left(r, r^{\prime}\right) \chi\left(r^{\prime}\right) \mathbf{D}\left(r^{\prime}\right) d V
$$

where $G\left(\boldsymbol{r}, \boldsymbol{r}^{\prime}\right)$ is three dimensional free-space Green function, its expression is:

$$
G\left(r, r^{\prime}\right)=\frac{e^{-j k\left|r-r^{\prime}\right|}}{4 \pi\left|r-r^{\prime}\right|}
$$

And $\chi(r)$ is defined as the difference between the dielectric permittivity and background, which expression is presented as following:

$$
\chi(r)=\frac{\varepsilon(r)-\varepsilon_{0}}{\varepsilon(r)}
$$

The dielectric object is covered by a cuboid and discretized by a regular grid, as the Figure 1 shows.

And the electric displacement field $\mathrm{D}(r)$ is divided into a set of basic functions.

$$
\mathbf{D}^{q}(r)=\sum_{n}^{N} d_{n}^{q} \phi_{n}^{q}
$$

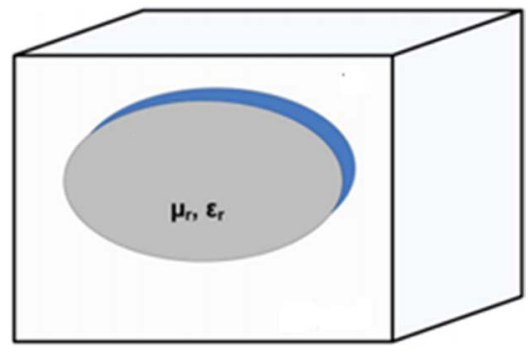

Figure 1. Arbitrary dielectric object embedded in a cuboid [7]. 
where $d_{n}$ is unknown, and $\phi_{n}^{q}$ is defined as the rooftop basic function on $\mathrm{x}, \mathrm{y}, \mathrm{z}$ directions respectively, and the superscript $q=\mathrm{x}, \mathrm{y}, \mathrm{z}$.

After substituting the representation of $\mathbf{A}(r)$ into Equation (1), the Galerkin testing formulation can be written as:

$$
\left\langle\varphi_{k}^{q}, \mathbf{E}^{i}(r)\right\rangle=\left\langle\varphi_{k}^{q}, \frac{\mathbf{D}\left(r^{\prime}\right)}{\varepsilon(r)}\right\rangle-\left\langle\varphi_{k}^{q},\left(k^{2}+\nabla \nabla \cdot\right) \frac{1}{\varepsilon_{0}} \iiint_{V} G\left(r, r^{\prime}\right) \chi\left(r^{\prime}\right) \mathbf{D}\left(r^{\prime}\right) d V\right\rangle
$$

Here, the operator $\langle.,$.$\rangle denotes the integration of inner product of two vec-$ tor functions.

Substituting the expansion for $\mathrm{D}(r)$ into Equation (6), above equation is represented as below:

$$
\begin{aligned}
\left\langle\varphi_{k}, \mathbf{E}^{i}(r)\right\rangle= & \sum_{n}\left[\left\langle\varphi_{k}^{q}, \frac{\varphi_{k}^{q}}{\varepsilon(r)}\right\rangle-\left\langle\varphi_{k}^{q}, k_{0}^{2} \iiint_{V} G\left(r, r^{\prime}\right) \chi\left(r^{\prime}\right) \varphi_{k}^{q}\left(r^{\prime}\right) d V\right\rangle\right. \\
& \left.+\left\langle\nabla \cdot \varphi_{k}^{q}, \iiint_{V} G\left(r, r^{\prime}\right) \nabla^{\prime} \cdot\left(\chi\left(r^{\prime}\right) \varphi_{k}^{q}\left(r^{\prime}\right)\right) d V\right\rangle\right] d_{n}
\end{aligned}
$$

For brevity, write the simplified Equation (7) as:

$$
\mathbf{B}_{q}=\mathbf{H}_{q} d_{n}^{q}+\mathbf{G}_{1} *\left(\mathbf{S}_{1} d_{n}^{q}\right)+\mathbf{G}_{2} *\left(\mathbf{S}_{2} d_{n}^{q}\right)
$$

In which, the property of rooftop basic function $\varphi_{m}(\boldsymbol{r})=\varphi_{0}\left(\boldsymbol{r}-\boldsymbol{r}_{m}\right)$ is applied. In Equation (8), $\mathbf{B}_{q}$ is the matrix decided by incident electric field. $\mathbf{G}_{1}$ and $\mathbf{G}_{2}$ are Toeplitz matrices. $\mathbf{S}_{1}$ and $\mathbf{S}_{2}$ are diagonal matrices with the parameters of the scatterers. The operator ${ }^{*}$ denotes the convolution. To solve Equation (8), Conjugate Gradient method is applied, in which Fast Fourier Transform is used for acceleration of convolution during iteration. The convolution in Equation (7) can be evaluated by using FFT efficiently as blow:

$$
\mathbf{G}_{1} *\left(\mathbf{S}_{1} d_{n}^{q}\right)=\operatorname{IFFT}\left(F F T\left(\mathbf{G}_{1}\right) \cdot F F T\left(\mathbf{S}_{1} d_{n}^{q}\right)\right)
$$

Therefore, the computational complexity per iteration remains order NlogN in CG algorithm [9] [10]. The above algorithm allows for the possibility of simulating the scattered field.

\section{Simulation}

In this section, we first validate the CG-FFT algorithm, and discuss the memory and time required, but also investigate the convergence of the CG method, which relates to material of object. Finally, the algorithm is applied for the simulation of a hemi-sphere dielectric radome.

Here we present a simulation of solid dielectric sphere to validate the CG-FFT algorithm. The radius is $0.5 \mathrm{~m}$, and the relative dielectric constant of the sphere equals to 4.0. In case of scatter, Radar Cross Section is the equivalent area seen by a radar, the bi-static RCS can be estimated as:

$$
\sigma=\lim _{r \rightarrow \infty} 4 \pi r^{2} \frac{\left|E^{s}\right|^{2}}{\left|E^{i n c}\right|^{2}}
$$


The incident wave is polarized in the $\mathrm{x}$-direction and propagating in $-\mathrm{z}$ direction. And incident frequency is $300 \mathrm{MHz}$. In post processing, the pitch angle $\theta$ range from 0 deg to $180 \mathrm{deg}$ and azimuth angle equals to $0 \mathrm{deg}$. The size of grid is $1 / 10$ of the wavelength in three dimensions. As Figure 2 shows, the bi-static RCS calculated by CG-FFT is corresponding to the result simulated by FEKO, which validates the correctness of the CG-FFT algorithm.

Take the dielectric sphere as an example, we discuss the consumption of the memory and the time spent during per iteration, which relates to the number of rooftop functions. Statistically, we present the memory and time vary according to the number of the rooftop functions. Additionally, the number of the rooftop functions equal to the product of numbers of rooftop functions in three directions $(q=\mathrm{x}, \mathrm{y}, \mathrm{z})$. The memory consumed is presented in Figure 3(a), which is approximately linear with the number of rooftop functions. And time consumption per iteration averagely is showed in Figure 3(b).

As Figure 3 shows, the memory consumed rises linearly with the number of rooftop functions. Because memory is almost used for the storage of the rooftop functions. Meanwhile, the time consumption per iteration increases nonlinearly.

Additionally, the efficiency is not only related to the consumption per iteration, but also related to the total number of iteration. We also discover that the total number of iteration has a relation to the relative dielectric constant of the object. The total number of iteration with different material property is presented in Table 1.

As Figure 4 shows, as the relative dielectric constant rises, the total number of iteration increases nonlinearly. Namely, if the relative dielectric constant of the electromagnetic object is closer to that of background, the less iteration is cost. For the object with large relative dielectric constant, the CG-FFT algorithm

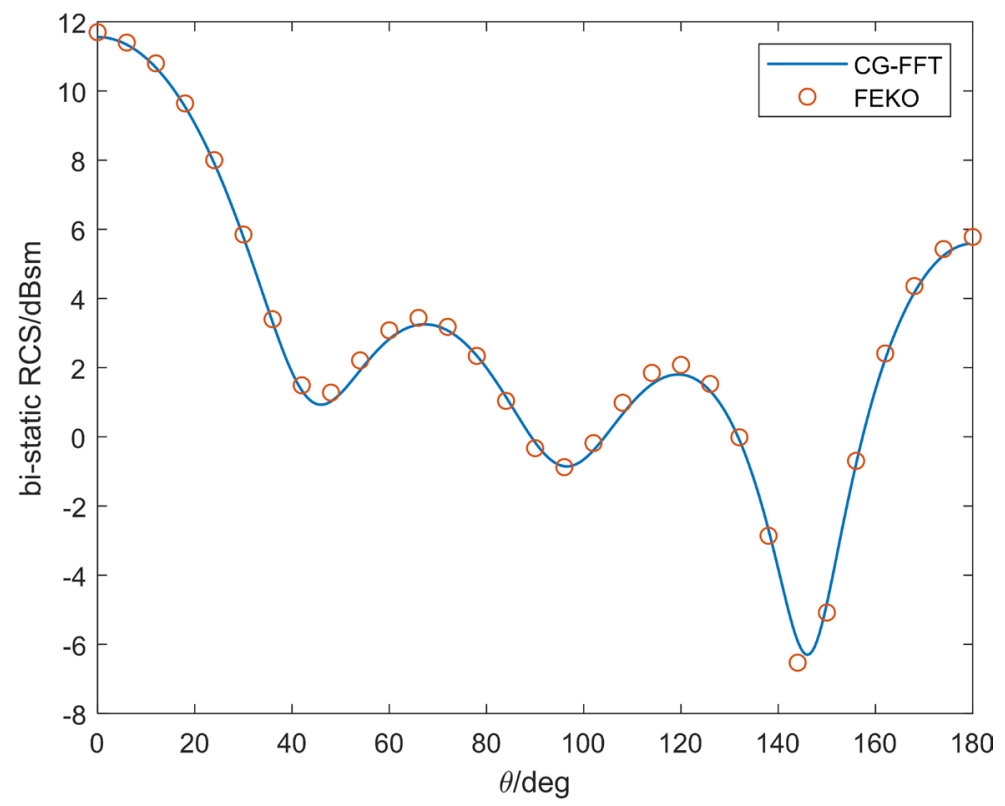

Figure 2. Bi-static RCS of dielectric sphere. 


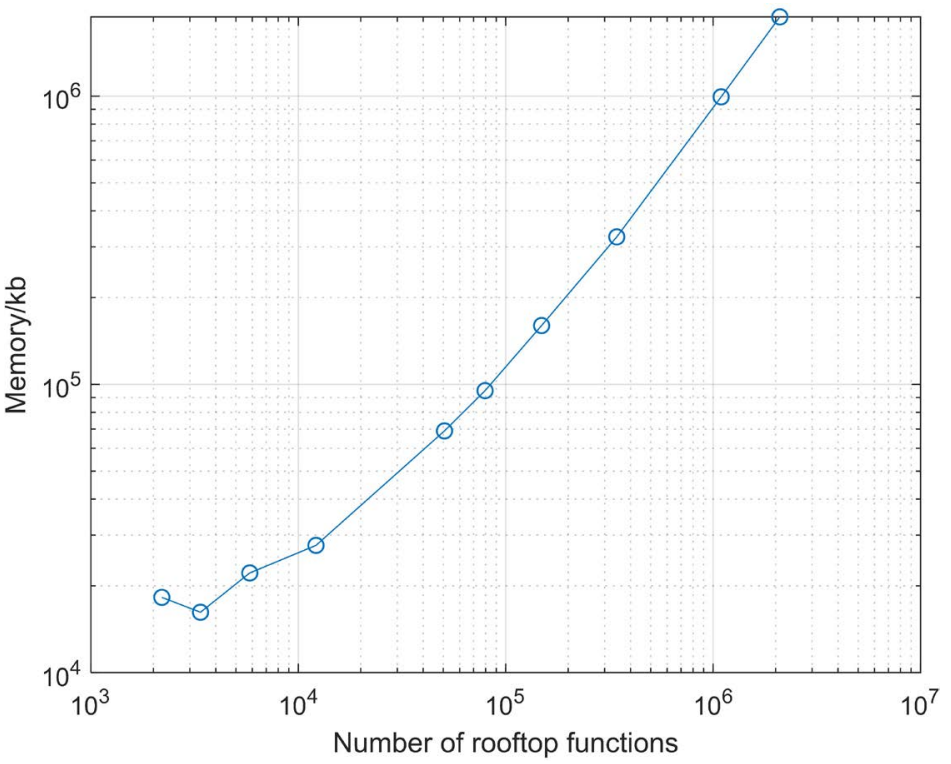

(a)

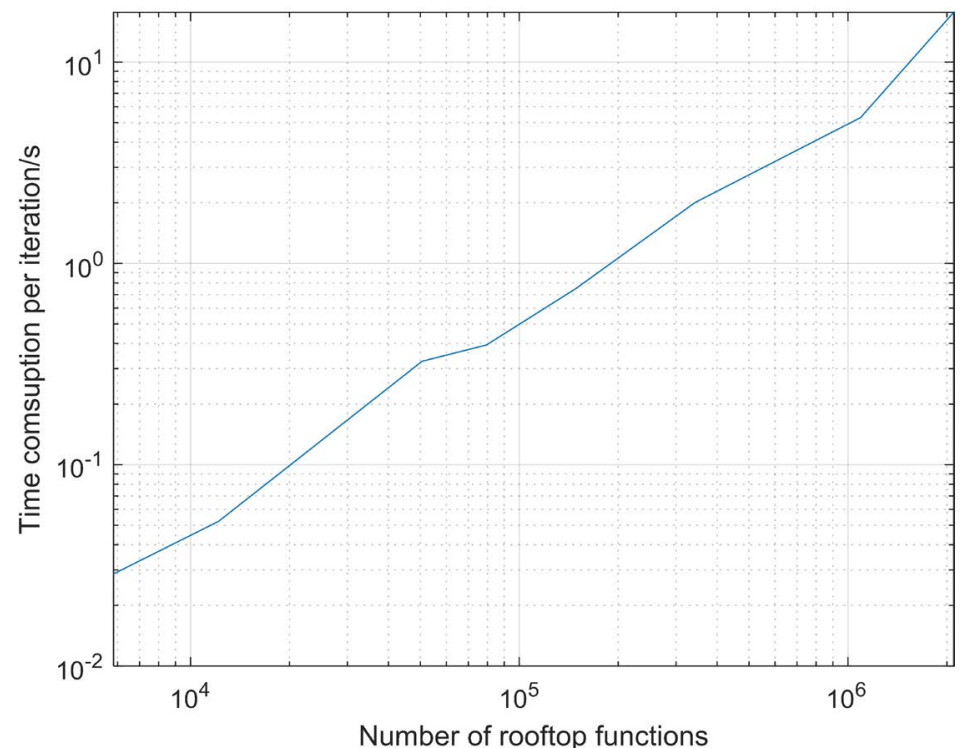

(b)

Figure 3. Time and memory consumption. (a) Memory consumed; (b) Time consumption per iteration averagely.

Table 1. Total number of iteration with different material property.

\begin{tabular}{cc}
\hline Relative dielectric constant & Total number of iteration \\
\hline 2 & 16 \\
3 & 42 \\
4 & 80 \\
5 & 110 \\
6 & 230 \\
7 & 198 \\
8 & 318 \\
\hline
\end{tabular}




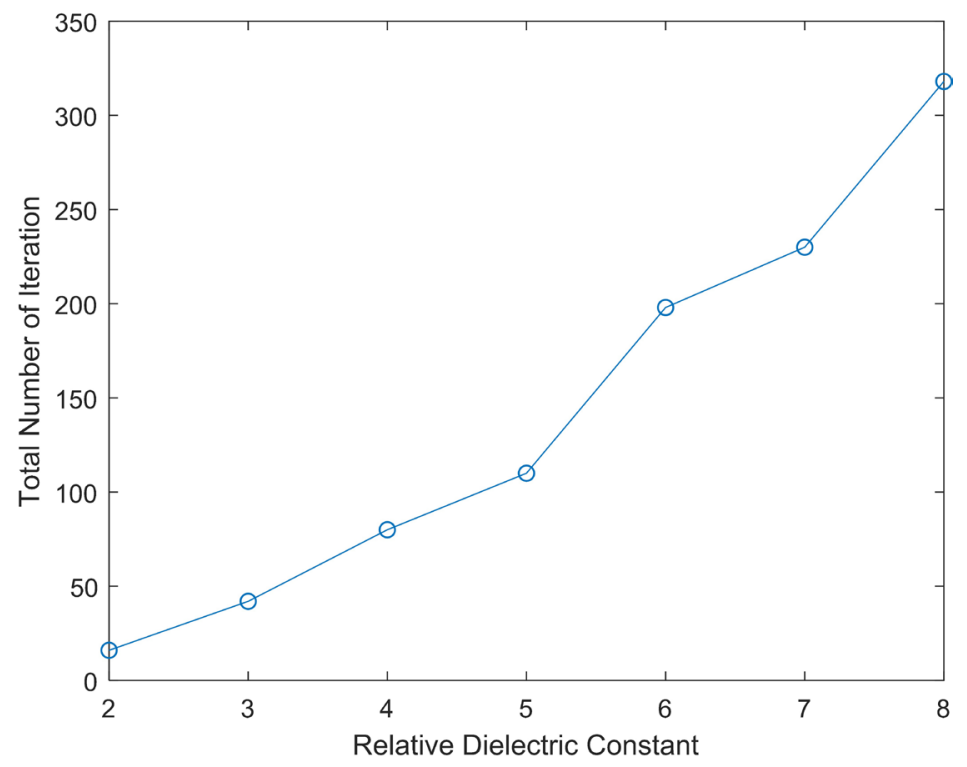

Figure 4. Total number of Iteration Increases nonlinearly.

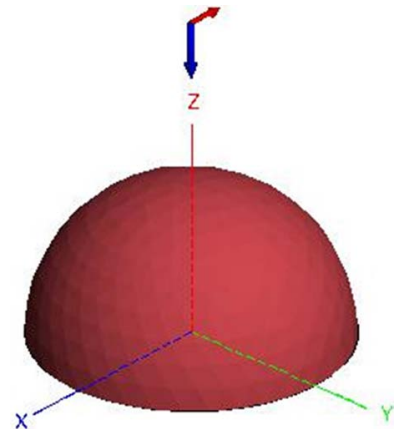

(a)

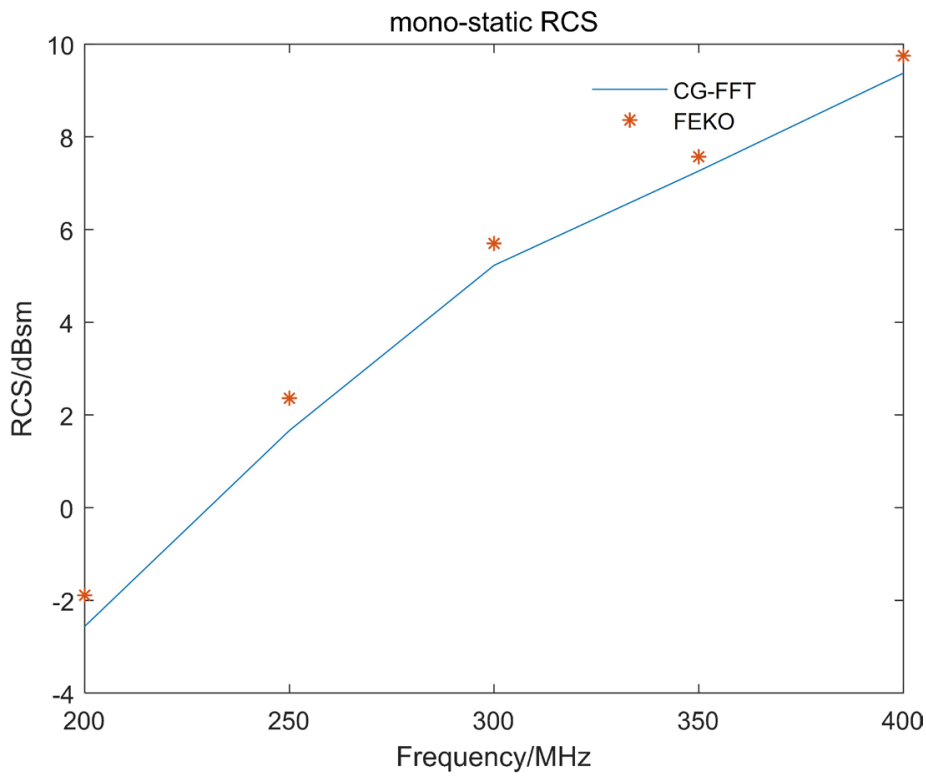

(b)

Figure 5. Simulation of the hemi-sphere dielectric radome. (a) Hemi-sphere dielectric radome; (b) Mono-static RCS of dielectric radome. 
performs not so good. However, radome is usually consisted of small relative dielectric constant, which means the algorithm is suitable for the simulation of radomes.

Next, a hemi-sphere dielectric radome is showed in Figure 5(a) and is simulated. The outer radius of radome is 1 meter. The thick is uniformly 10 micrometer. The relative dielectric constant equals 4.0. The incident wave is polarized in the $\mathrm{x}$-direction and propagating in $-\mathrm{z}$ direction. The mono-static RCS of dielectric radome computed by CG-FFT and that from commercial software FEKO is showed in Figure 5(b), which validates the CG-FFT algorithm. The frequency ranges from $200 \mathrm{MHz}$ to $400 \mathrm{MHz}$.

\section{Conclusion}

In this paper, the CG-FFT algorithm is applied for the simulation of the dielectric radome. In this algorithm, the Fast Fourier Transform is combined with Conjugate Gradient algorithm, in which the computational complexity of per iteration remains order $\operatorname{Nog} \mathrm{N}$. The result above indicates its feasibility for simulation of dielectric radomes.

\section{Conflicts of Interest}

The authors declare no conflicts of interest regarding the publication of this paper.

\section{References}

[1] Liu, N., Sheng, X.J., Zhang, C.B., et al. (2017) Design of Frequency Selective Surface Structure with High Angular Stability for Radome Application. IEEE Antennas \& Wireless Propagation Letters, PP, 1-1. https://doi.org/10.1109/lawp.2017.2778078

[2] Wahab, M. (2009) Radar Radome and Its Design Considerations. International Conference on Instrumentation, Communications, Information Technology, \& Biomedical Engineering. https://doi.org/10.1109/icici-bme.2009.5417229

[3] Narayan, S., Shamala, J.B., Nair, R.U., et al. (2012) Electromagnetic Performance Analysis of Novel Multi-Band Metamaterial FSS for Millimeter Wave Radome Applications. Computers, Materials \& Continua, 31, 1-15.

[4] Moreno, J., et al. (2013) Analysis and Design of Antenna Radomes. 2013 IEEE International Conference on Microwaves, Communications, Antennas and Electronic Systems IEEE. https://doi.org/10.1109/comcas.2013.6685299

[5] Sadiku, M.N.O., Shadare, A.E. and Musa, S.M. (2017) Computational Electromagnetics. International Journal of Engineering Research, 6, 414-416.

[6] Gan, H. and Chew, W.C. (1995) Fast Computation of 3D Inhomogeneous Scattered Field Using a Discrete BCG-FFT Algorithm. IEEE Antennas and Propagation Society International Symposium. https://doi.org/10.1109/aps.1995.530868

[7] Fan, G.X. and Liu, Q.H. (2001) The CGFFT Method with a Discontinuous FFT Algorithm. Microwave \& Optical Technology Letters, 29, 47-49. https://doi.org/10.1002/mop.1079

[8] Zwanborn, A.P.M. and van den Berg, P.M. (1991) A Weak Form of the Conjugate Gradient FFT Method for Plate Problems. IEEE Transactions on Antennas \& Prop- 
agation, 39, 224-228. https://doi.org/10.1109/8.68186

[9] Zhang, S.L. (1997) GPBi-CG: Generalized Product-Type Methods Based on Bi-CG for Solving Nonsymmetric Linear Systems. SIAM Journal on Scientific Computing, 18. https://doi.org/10.1137/s1064827592236313

[10] Yu, Z., Zhang, W. and Liu, Q.H. (2014) A Mixed-Order Stabilized Bi-Conjugate Gradient FFT Method for Magnetodielectric Objects. IEEE Transactions on Antennas and Propagation, 62, 5647-5655. https://doi.org/10.1109/tap.2014.2352634 